Check for

updates

Cite as

Nano-Micro Lett.

(2021) 13:194

Received: 12 May 2021

Accepted: 11 July 2021

Published online: 14 September 2021

(C) The Author(s) 2021

\section{High-Efficiency Wastewater Purification System Based on Coupled Photoelectric-Catalytic Action Provided by Triboelectric Nanogenerator}

Shen Shen ${ }^{1,2}$, Jiajia $\mathrm{Fu}^{2}, \mathrm{Jia} \mathrm{Yi}^{1}$, Liyun $\mathrm{Ma}^{1}$, Feifan Sheng ${ }^{1}$, Chengyu $\mathrm{Li}^{1}$, Tingting Wang ${ }^{1}$, Chuan Ning ${ }^{1,3}$, Hongbo Wang ${ }^{2}$, Kai Dong ${ }^{1,3}{ }^{凶}$, Zhong Lin Wang ${ }^{1,3,4} \bowtie$

\title{
HIGHLIGHTS
}

- Triboelectric nanogenerator (TENG)/carbon dots-TNs supported over three-dimensional graphene aerogel (3DGA@CDs-TNs) system was constructed.

- TENG and 3DGA@CDs-TNs present excellent degradation activity toward brilliant green $(88.26 \%, 40 \mathrm{~min})$ and direct blue 5B $(89.6 \%$, $90 \mathrm{~min})$.

- The coupling interaction of photocatalysis and TENG leads to an enhanced removal ability.

- The degradation pathways for brilliant green and direct blue 5B are proposed.

ABSTRACT It is of great importance to explore a creative route to improve the degradation efficiency of organic pollutants in wastewater. Herein, we construct a unique hybrid system by combining self-powered triboelectric nanogenerator (TENG) with carbon dots- $\mathrm{TiO}_{2}$ sheets doped three-dimensional graphene oxide photocatalyst (3DGA@CDs-TNs), which can significantly enhance the degradation efficiency of brilliant green (BG) and direct blue 5B (DB) owing to the powerful interaction of TENG and 3DGA@CDs-TNs photocatalyst. The power output of TENG can be applied for wastewater purification directly, which exhibits a selfpowered electrocatalytic technology. Furthermore, the results also verify that TENG can replace conventional electric catalyst to remove pollutants effectively from wastewater without any consumption. Subsequently, the unstable fragments and the plausible removal pathways of the two pol-

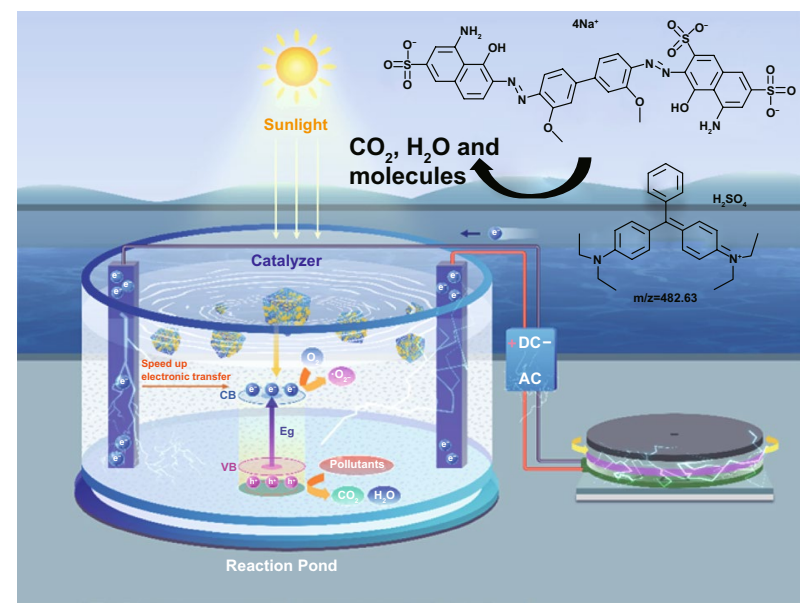
lutants are proposed. Our work sheds light on the development of efficient and sustainable TENG/photocatalyst system, opening up new opportunities and possibilities for comprehensive utilization of random energy.

KEYWORDS Triboelectric nanogenerators; Self-powered; 3DGA@CDs-TNs photocatalyst; Hybrid system; Wastewater purification

Shen Shen and Jiajia Fu have contributed equally to this work.

$\bowtie$ Hongbo Wang, wxwanghb@163.com; Kai Dong, dongkai@binn.cas.cn; Zhong Lin Wang, zhong.wang@mse.gatech.edu

1 CAS Center for Excellence in Nanoscience, Beijing Key Laboratory of Micro-Nano Energy and Sensor, Beijing Institute of Nanoenergy and Nanosystems, Chinese Academy of Sciences, Beijing 100083, People's Republic of China

2 Jiangsu Engineering Technology Research Centre for Functional Textiles, Jiangnan University, No.1800 Lihu Avenue, Wuxi, People's Republic of China

3 School of Nanoscience and Technology, University of Chinese Academy of Sciences, Beijing 100049, People's Republic of China

4 School of Material Science and Engineering, Georgia Institute of Technology, Atlanta, GA 30332-0245, USA 


\section{Introduction}

According to the World Wildlife Fund (WWF) and UNESCO (The United Nations Educational, Scientific and Cultural Organization), a large portion of water source has changed toward deterioration situation. Currently, under the water consumption trend, the two organizations have evaluated that the world population could suffer from water shortages by 2025 [1-3]. For wastewater, organic dyes created during the industries untreated emission are a major issue not only owning to the hazardous effect on aquatic quality but also due to their persistence, toxicity as well as carcinogenicity [4-6]. In particular, dyes can cause hindrance to the sunlight penetration and reduction in dissolved oxygen, which disturb ecological balance. For these reasons, removal and lowering of toxicity of organic dyes from industrial water are impending $[7,8]$.

Threatened by the scarcity of worldwide energy supply and ever-growing environmental crisis, an enormous challenge aiming to achieve better degradation efficiency of organic pollutants and high utilization of clean energy (blue energy, water energy, and wind energy) is extremely urgent [9-11]. Photocatalytic technique is a compelling strategy for aquatic and environmental remediation owning to its relatively low cost and robust chemical stability with no secondary pollution $[12,13]$. Solar energy can stimulate catalysts to generate photoelectron holes and produce active radicals $\left(\mathrm{OH}, \mathrm{O}_{2}^{-}\right)$, which can destroy or oxidate organic matters [14-16]. Howbeit, due to the low solar energy utilization associated with easy recombination of $\mathrm{e}^{-}-\mathrm{h}^{+}$pairs, the degradation efficiency of photocatalyst is limited [17-19]. Most important, the solar energy is not always available in environment, which makes photocatalystic degradation is not occur in the darkness. For this situation, construction of hybrids system is a key factor in improving overall efficiency [20-22]. Among various hybrids, photoelectric catalysis exhibits superior energy conversion efficiency and outstanding catalytic capacity [23-25]. In particular, owning to the interactions between the two routes, coupling of electrocatalysis with photocatalysis can enhance the amount of charge carriers and hinder the recombination of photogenerated electron-hole pairs [26-28]. However, traditional electrocatalysis confronts several defects, including large electricity consumption, harsh reaction conditions, and external power sources, which severely restrict its applications.

Triboelectric nanogenerator (TENG) with the ability of sustainable energy harvesting can provide an effective method for directly addressing the above issues [29-34]. As an alternative candidate, TENG may replace traditional electrocatalysis due to self-powered without any consumption and has no dependence on environmental conditions [35-38]. Although some related researches have been explored, there is no report about a system that consists of TENG and photocatalytic for purifying wastewater. The purpose of employing TENG is to use self-powered instead of electrocatalysis or other technologies to purify pollutants from wastewater [39-41]. TENG can capture and convert ambient motions into electrical energy based on the combination of triboelectrification and electrostatic induction, which has sparked extensive research for scavenging power [42-47]. Recently, TENG, as a promising self-powered manner toward harnessing blue energy, can be used for splitting water [48-50] and treating air pollutants [51]. Notably, energy from wind, solar and ocean waves has the potential to be integrated with electrical power grids to meet wastewater treatment requirements thanks to the infinite availability of renewable energy in nature [52].

Based on the above discussion, we construct a new working system to remove organic pollutants by combining TENG with photocatalysis technique. Carbon quantum dots- $\mathrm{TiO}_{2}$ sheets (CDs-TNs) catalysts were introduced into 3DGA structure (3DGA@CDs-TNs photocatalyst) by the addition of CDs-TNs during the hydrothermal synthesis of 3DGA. The interaction effect of 3DGA@CDs-TNs photocatalyst and TENG is analyzed by photoelectrocatalytic experiments. The pollutant degradation performance of TENG/3DGA@CDs-TNs system is superior to that of TENG and 3DGA@CDs-TNs photocatalyst alone, signifying TENG/3DGA@CDs-TNs has the potential used for wastewater remediation. In addition, possible pathways for the degradation of $\mathrm{BG}$ and $\mathrm{DB}$ are systematically proposed via the identification of fragments based on the identification of process fragments via liquid chromatography-tandem mass spectrometry (LC-MS/MS). Finally, the underlying degradation mechanism of the TENG/3DGA@ CDs-TNs is also clarified. 


\section{Materials and Methods}

\subsection{Materials}

Graphite powder (99.8\%), tetrabutyl titanate (TBOT), Congo red, and citric acid were provided by Aladdin Reagent Company. Sodium nitrate $\left(\mathrm{NaNO}_{3}\right)$, sulfuric acid $\left(\mathrm{H}_{2} \mathrm{SO}_{4}\right)$, potassium permanganate $\left(\mathrm{KMnO}_{4}\right)$, hydrochloric acid $(\mathrm{HCl})$, hydrofluoric acid (HF), sodium hydroxide $(\mathrm{NaOH})$, hydrogen peroxide $\left(\mathrm{H}_{2} \mathrm{O}_{2}\right)$, brilliant Green $(\mathrm{BR})$, and direct sky blue 5B (DB) were purchased from Sinopharm Chemical Reagent Co., Ltd, China (SCRC). Ammonium hydroxide was supplied by J\&K Scientific Ltd. DI water from ULUPURE pure ultrapure water system was used in experiments.

\subsection{Preparation of CDs-TNs}

$\mathrm{TiO}_{2}$ were prepared by hydrothermal method as depicted previously [53]. Typically, $3 \mathrm{~mL}$ of HF was added into $25 \mathrm{~mL}$ of TBOT with stirring. The mixture was placed into a Teflon-lined autoclave and kept at $180{ }^{\circ} \mathrm{C}$ for $24 \mathrm{~h}$. The products were collected and filtered by $\mathrm{NaOH}$ and DI water, and then by an oven. $1.051 \mathrm{~g}$ of citric acid was dissolve into $25 \mathrm{~mL}$ of DI water, and the $\mathrm{pH}$ value of solution was adjusted to 6 by using $\mathrm{NH}_{4} \mathrm{OH}$. After that, $0.4 \mathrm{~g}$ of TNs were added into the above mixture. The solution was sealed in a Teflon-lined autoclave at $160{ }^{\circ} \mathrm{C}$ for $4 \mathrm{~h}$. Afterward, the precipitates were collected by centrifugation and washed with DI water and then dried at $60{ }^{\circ} \mathrm{C}$ for $12 \mathrm{~h}$.

\subsection{Preparation of 3DGA@CDs-TNs Photocatalyst}

Graphene oxide (GO) was synthesized through the modified Hummers' method [54]. In a briefly approach for the synthesis of 3DGA@CDs-TNs photocatalyst, $20 \mathrm{mg}$ of asprepared CDs-TNs was added into $20 \mathrm{~mL}$ of $\mathrm{GO}$ dispersion (6 $\left.\mathrm{mg} \mathrm{mL}^{-1}\right)$ to obtain a uniform mixture. The mixture was sonicated and stirred for $1 \mathrm{~h}$. Then the mixture was transferred into $100 \mathrm{~mL}$ Teflon-lined autoclave and maintained at $180{ }^{\circ} \mathrm{C}$ for $12 \mathrm{~h}$ to synthetize 3DGA@CDs-TNs photocatalyst. Finally, the prepared 3DGA@CDs-TNs photocatalyst was purified with DI water and freeze-dried for $12 \mathrm{~h}$. The 3DGA aerogel was synthesized via the same approach as described above without adding CDs-TNs catalysts.

\subsection{Fabrication of TENG}

The framework of the TENG is separated into two components: a rotator and a stator. For the stator: (1) An acrylic plate with the thickness of $3 \mathrm{~mm}$ and the diameter of $20 \mathrm{~cm}$ was employed to form a square-shaped substrate using a laser cutter. (2) A layer of soft foam was pasted on the acrylic acts as buffer layer. (3) A layer of conductive fabric was adhered onto the soft foam. The conductive fabric as electrode was patterned using a laser cutter (diameter: $14.4 \mathrm{~cm}$ ) and had complementary as well as separated sectors. (4) A layer of PTFE as one of the triboelectric layer was pasted on the conductive fabric. For the rotator: (1) A disk-shaped acrylic substrate (diameter: $14.4 \mathrm{~cm}$, thickness: $3 \mathrm{~mm}$ ) was shaped through a laser cutter, which consisted of radially arrayed sectors with a central angle of $18^{\circ}$. (2) A copper layer was stuck on the side of acrylic substrate as friction layer, which possesses the same shape as that of one electrode.

\subsection{Characterization}

Thechemical states of as-prepared samples were characterized by an X-ray diffraction (XRD, Bruker AXS) under $\mathrm{Cu} \mathrm{K} \alpha$ radiation with diffraction angle of $10^{\circ}$ to $90^{\circ}$. The microstructures and morphological structures were captured through scanning electron microscopy (SEM) by su1510 microscope and transmission electron microscopy (TEM) by JEM-2010 electron microscope (JEOL). The electrochemical impedance spectroscopy (EIS) and photocurrent response measurements were performed by using Zennium PP211 electrochemical workstation. The output voltage and current of the TENG were monitored by an electrometer (Keithley 6514).

The LC-MS technique was employed to identify the decomposed intermediates and final products of BG and TC (Waters, USA); BEH C18 column $(1.7 \mu \mathrm{m}, 2.1 \times 150 \mathrm{~mm})$. The detailed works are given in supporting information.

\subsection{Organic Pollutants Degradation}

Photocatalytic removal experiment: the visible light irradiation was achieved by a $500 \mathrm{~W}$ (XO-CHX-ID) Xe lamp with a $420 \mathrm{~nm}$ cutoff filter. Briefly, $10 \mathrm{mg}$ of as-prepared catalysts 
was suspended in $30 \mathrm{~mL}$ of BG (5 ppm) and DB (20 ppm) aqueous solution, and the solution was stirred in the darkness to ensure the adsorption-desorption equilibrium. At preset intervals, the reaction solution was collected and filtered to remove catalysts. The absorbance of BG and DB in supernatant was examined by UV-Vis spectrophotometer.

Self-powered integration system for pollutants removal: all the experiments for degrading $\mathrm{BG}$ and $\mathrm{DB}$ were carried out in a breaker containing $30 \mathrm{~mL}$ solution of BG and DB. $10 \%$ of $\mathrm{NaCl}$ solution was selected as electrolyte. Graphite and iron acting as cathode and anode were immersed into the reaction container. A rectifying bridge was used to convert the alternating current to direct current.

\section{Results and Discussion}

\subsection{Structure and Output Performance of TENG}

The multifunctional structure of TENG is displayed in Fig. 1a, which is mainly composed of two parts: a rotator (a)

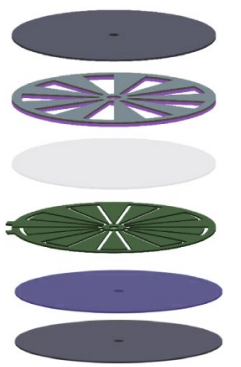

(b)

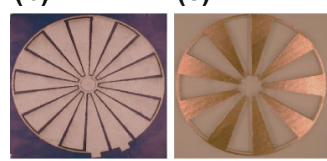

(c) (d)

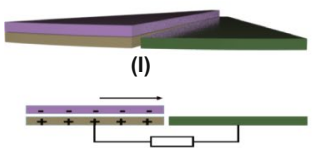

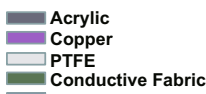
Copper
PTFE
Conductive Fabric Foam

(e)

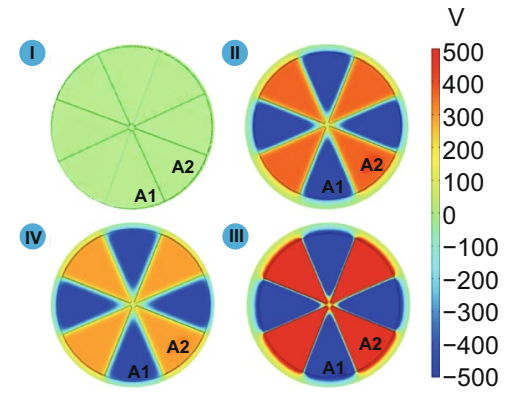

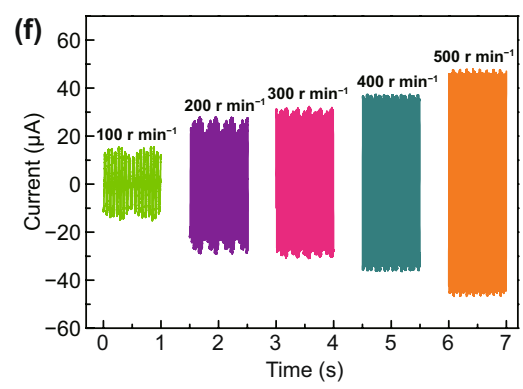
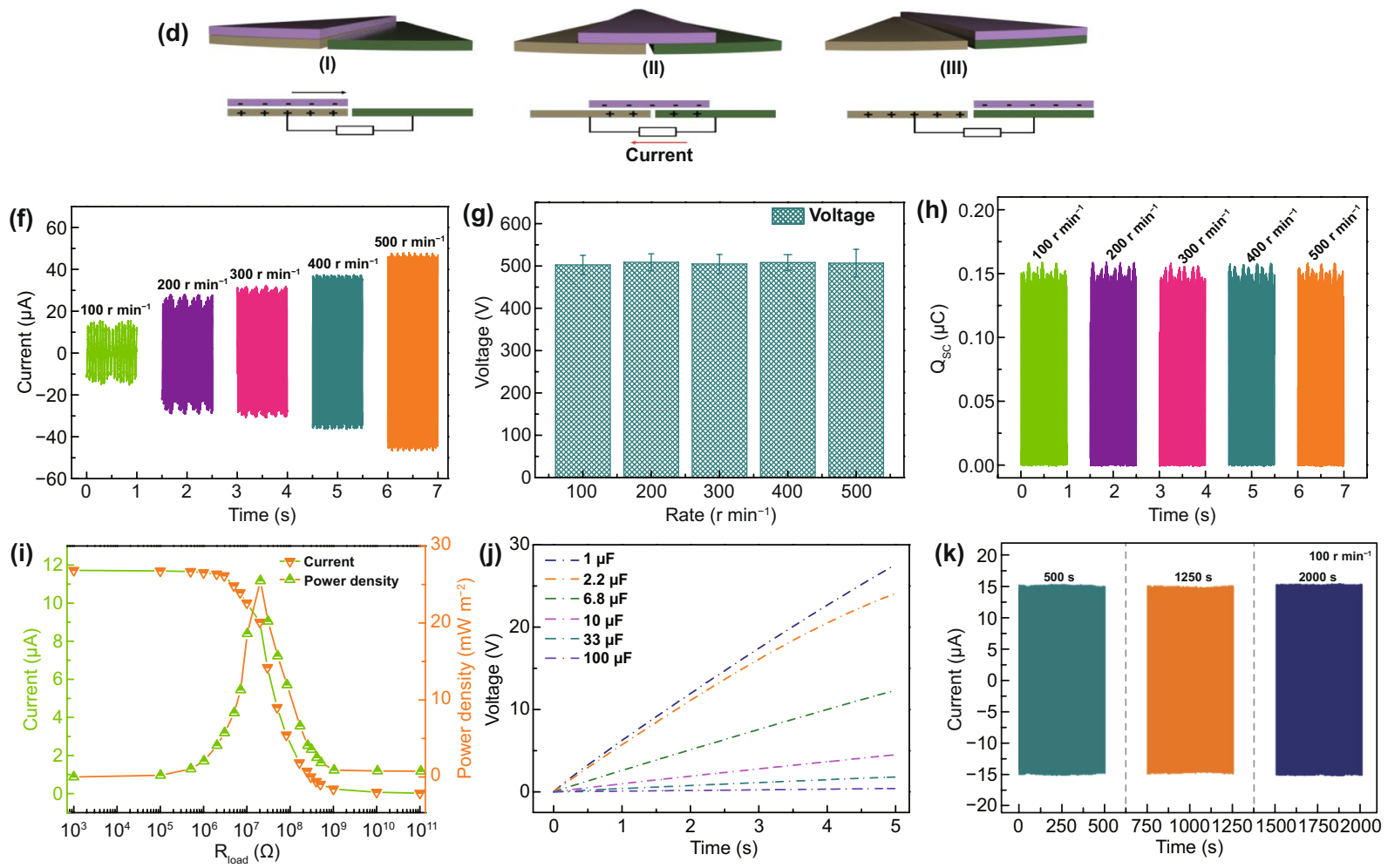

Fig. 1 Schematic diagram. a A schematic illustration of the functional components of multifunctional TENG. A photograph of b stator and c rotator. The working principles of TENG with the freestanding module: $\mathbf{d}$ schematic working principle of TENG (I-III) and e the simulated potential distributions for TENG at three different rolling displacements by COMSOL software. Performance of TENG. f Short-circuit current, $\mathbf{g}$ open-circuit Voltage, $\mathbf{h}$ short-circuit charge quantity of TENG at different rotation rates. $\mathbf{i}$ Capacitor charging ability of the TENG under 100 $\mathrm{r} \mathrm{min}^{-1}$. j Current and peak power of the TENG measured with different external load resistances under $100 \mathrm{r}$ min $^{-1}$. k Stability of open-circuit voltage over 16,000 cycles 
and a stator. The rotator consists of an acrylic sheet with uniformly distributed 9 of $\mathrm{Cu}$ layers. Each of radial array sectors has a length of $7.2 \mathrm{~cm}$ and central angle of $18^{\circ}$. And the $\mathrm{Cu}$ layer acts as contact-triboelectrification surface. For the stator, it can be divided into four parts: a layer of polytetrafluoroethylene film (PTFE) as an electrification material, a layer of conductive fabric deposited on the soft foam as the electrodes and an acrylic sheet as the underlying substrate. In particular, the electrode layer is constituted by two adjacent complementary electrode frameworks which are separated by gaps. In addition, the photographs of the rotator and the stator are presented in Fig. 1b, c. The working mechanism of TENG is illustrated in Fig. 1d. In the original state, owning to the different triboelectric polarities of the two contact materials, positive and negative charges can be produced on the $\mathrm{Cu}$ surface and PTFE film, respectively, after triboelectrification (step i). Once the rotator sweeps across the stator, charges are injected from $\mathrm{Cu}$ layer to the PTFE film due to the conservation of charge (step ii). Once the $\mathrm{Cu}$ electrode overlaps the electrode $\mathrm{B}$, all the electrons are flowed to the electrode B (step iii). As visualized in Fig. 1e, numerical calculation results further demonstrate the electrostatic potential difference of TENG in the contacting state and separating state.

The electrical output performance of TENG including open-circuit voltage $\left(V_{\mathrm{OC}}\right)$, short-circuit current $\left(I_{\mathrm{SC}}\right)$, and short-circuit charge transfer $\left(Q_{\mathrm{SC}}\right)$ with variable rotating speeds is presented in Fig. 1f-h. The $I_{\mathrm{SC}}$ of TENG is gradually enhanced with the increase in the rotating speed. As the rotating speed increases from 100 to $500 \mathrm{r} \mathrm{min}^{-1}$, the $I_{\mathrm{SC}}$ increases from 15.6 to $48.2 \mu \mathrm{A}$ (Fig. 1f), suggesting that the higher rotating speed can enhance the output current. The $V_{\mathrm{OC}}$ with different rotating speeds are shown in Fig. $1 \mathrm{~g}$. As depicted, the $V_{\mathrm{OC}}$ is saturated at about $510 \mathrm{~V}$. Moreover, with increasing the rotating speed, no obvious change of the $V_{\mathrm{OC}}$ is found, which indicates that the increase in the rotating speed has no effect on the $V_{\mathrm{OC}}$. In addition, there is no enhance for $Q_{\mathrm{SC}}$ with the speed accelerates, and a steady value at about $0.153 \mu \mathrm{C}$ is observed (Fig. 1h).

To evaluate the output power of TENG, the output current was tested by connecting it with different external resistances (Fig. 1i). At a rotator rate of $100 \mathrm{r} \mathrm{min}^{-1}$, the output current drops with the external resistances increase. The output power achieves the maximum peak of $25.48 \mathrm{~mW} \mathrm{~m}^{-2}$ rapidly at a resistance of $20 \mathrm{M} \Omega$. Furthermore, a series of capacitors $(1-100 \mu \mathrm{F})$ are employed to demonstrate the charging capability of TENG. As shown in Fig. 1j, the capacitor $(1 \mu \mathrm{F})$ is charged to $27.8 \mathrm{~V}$ in $5 \mathrm{~s}$ under the rotator rate of $100 \mathrm{r} \mathrm{min}^{-1}$. With increasing the capacitance, the voltage of capacitors clearly decreases and a sluggish charging process is occurred.

Robust stability is a critical concern for TENG in practical application [55]. Thus, the output performance of TENG was measured under $100 \mathrm{r} \mathrm{min}^{-1}$ during 2000s consecutive rotation. As shown in Fig. 1k, the voltage of TENG exhibits a stable trend and an unobservable variation, proving a favorable stability of TENG.

\subsection{Structure and Electrochemical Performance of 3DGA@CDs-TNs Photocatalyst}

Figure 2a shows the synthesis of 3DGA@CDs-TNs photocatalyst. The microstructures of samples were captured by SEM and TEM. It is shown that GO has smooth surface with a 2D structure. As displayed in the insets of Fig. 2b, c, the 3DGA@CDs-TNs photocatalyst appears smaller than the blank 3DGA. Simultaneously, the structure of 3DGA and 3DGA@CDs-TNs photocatalyst shows the large appearance area and presents spatial network with cross-linked pores composing by randomly dispersed GO nanosheets. Figure $2 \mathrm{e}$ displays an ultrathin sheets and irregular creased of GO. The TEM image of 3DGA shows a folding structure (Fig. 2f). Figure 2g exhibits the TEM image of 3DGA@CDs-TNs photocatalyst, from which it can be observed that CDs-TNs catalysts are well dispersed on the surface of 3DGA. In addition, the synthesized CDs are spherical and well dispersed (Fig. S1a). Meanwhile, the TEM image of $0.2 \mathrm{CDs} / \mathrm{TNs}$ (Fig. S1b) exhibits CDs uniformly distributed on the TNs surface. EDS mapping images (Fig. 2h-1) of 3DGA@CDs-TNs photocatalyst further demonstrate the existence of $\mathrm{C}, \mathrm{N}, \mathrm{O}, \mathrm{Ti}$ elements in 3DGA@CDs-TNs photocatalyst.

The survey XPS spectrum of 3DGA@CDs-TNs photocatalyst further reveals the presence of $\mathrm{C}, \mathrm{N}, \mathrm{O}$, Ti elements. In addition, the respective atomic content is $69.65 \%, 1.18 \%$, $24.38 \%$, and $4.79 \%$ (Fig. $2 \mathrm{~m}$ and Table S1). For the C 1s region (Fig. 2n), the signal can be divided into three peaks at $284,285.1$, and $288.7 \mathrm{eV}$, corresponding to $\mathrm{C}=\mathrm{C} / \mathrm{C}-\mathrm{C}$, $\mathrm{C}-\mathrm{Ti}-\mathrm{O}$, and $\mathrm{C}=\mathrm{O}$. According to the Fig. 2o, the $\mathrm{O} 1 \mathrm{~s}$ band is fitted to four peaks at $530.2,531.3$, and $532.3 \mathrm{eV}$, which are related to the occurrence of $\mathrm{C}=\mathrm{O}, \mathrm{C}-\mathrm{OH}$, and 


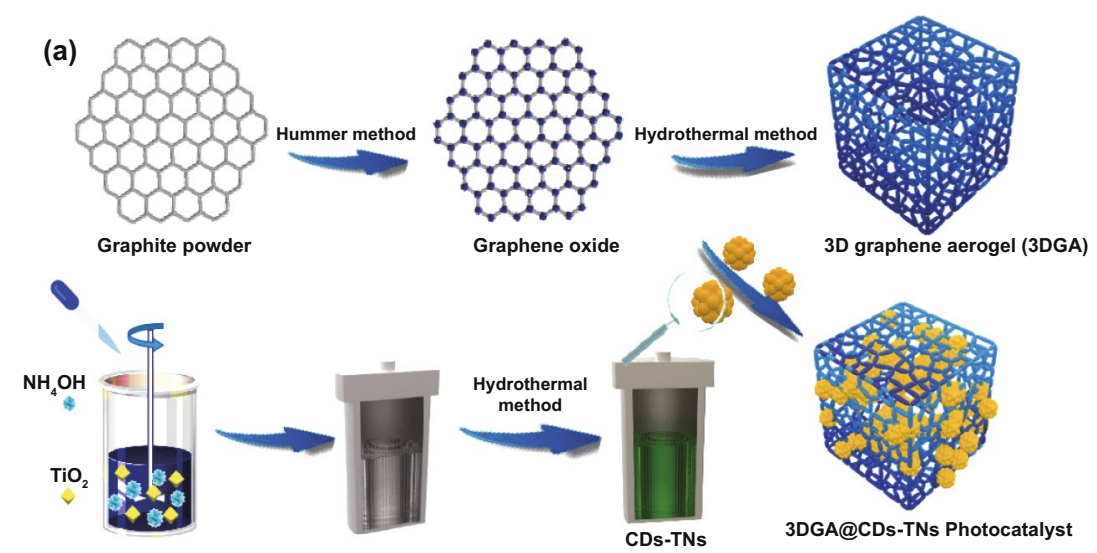

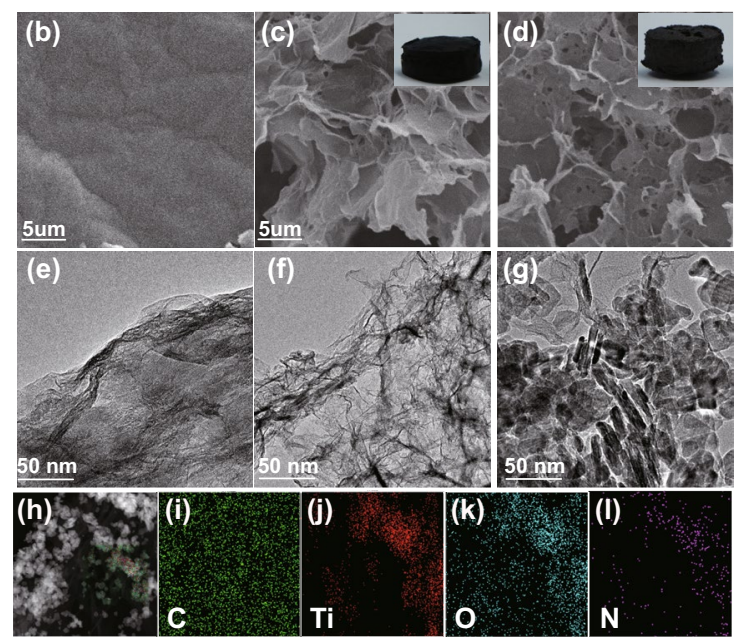

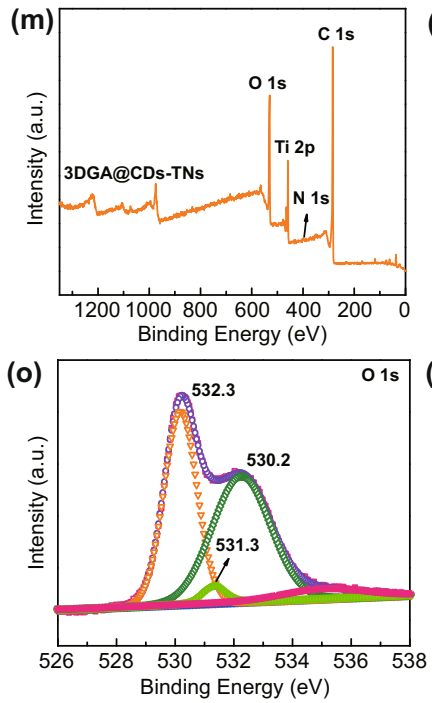

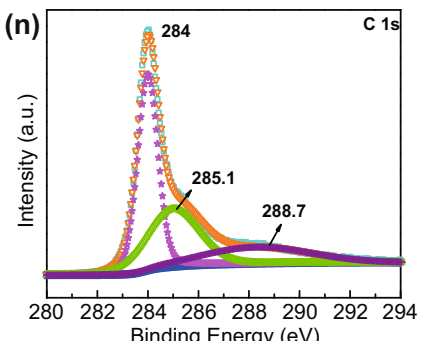

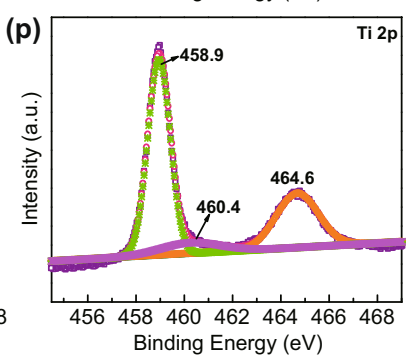

Fig. 2 a Schematic showing the synthesis of 3DGA@CDs-TNs photocatalyst. SEM images of b GO, c 3DGA inset: the picture of 3DGA; and d3DGA@CDs-TNs, inset: the picture of 3DGA@CDs-TNs. TEM image of e GO, f 3DGA, and 3DGA@CDs-TNs g, and corresponding EDS. h-l mapping images for survey, C, N, O, and Ti in 3DGA@CDs-TNs photocatalyst. XPS spectra of 3DGA@CDs-TNs photocatalyst. m Survey spectrum, n C 1 s, o O 1s, and $\mathbf{p}$ Ti $2 p$

O-Ti-O. Figure 2p shows the Ti 2p carves of 3DGA@CDsTNs photocatalyst, which can be deconvoluted into three peaks $(458.9,460.4$, and $464.6 \mathrm{eV})$ including two peaks of Ti 2 p3/2 and the peak of Ti 2p1/2. XPS results confirm the electronic interaction between CDs-TNs and 3DGA in 3DGA@CDs-TNs photocatalyst.

Figure $3 \mathrm{a}, \mathrm{b}$ illustrates the XRD patterns of samples. The distinct diffraction peak at $30.5^{\circ}$ for CDs is index to (200) disordered graphite-like species. For TNs and CDs/ TNs composites, all characteristic peaks from $25.28^{\circ}$ to $75.03^{\circ}$ correspond to the crystal planes of anatase $\mathrm{TiO}_{2}$ (PDF No. 00-021-1272). Nevertheless, for CDs-TNs composites, no characteristic diffraction for CDs is observed, owing to the limited content of CDs in the latter. GO sample shows the distinct peak at $7.7^{\circ}$, owing to the (002) plane of GO. After hydrothermal route, the peak of GO almost disappears and a broad distinct peak of 3DGA occurs at $16.6^{\circ}$, indicating that $\mathrm{GO}$ is converted into $\mathrm{rGO}$ successfully by hydrothermal process. For 3DGA@CDsTNs photocatalyst, the peaks are dramatically shifting to high angle due to the existence of CDs-TNs catalysts on 3DGA structure, which expand the interlayer spacing. Moreover, Fig. S2 shows the XRD patterns of samples after reaction. It can be seen that there are no noticeable changes in the crystal structure for 3DGA@CDs-TNs before and after degrading, which is further verifies the robust stability of 3DGA@CDs-TNs. 

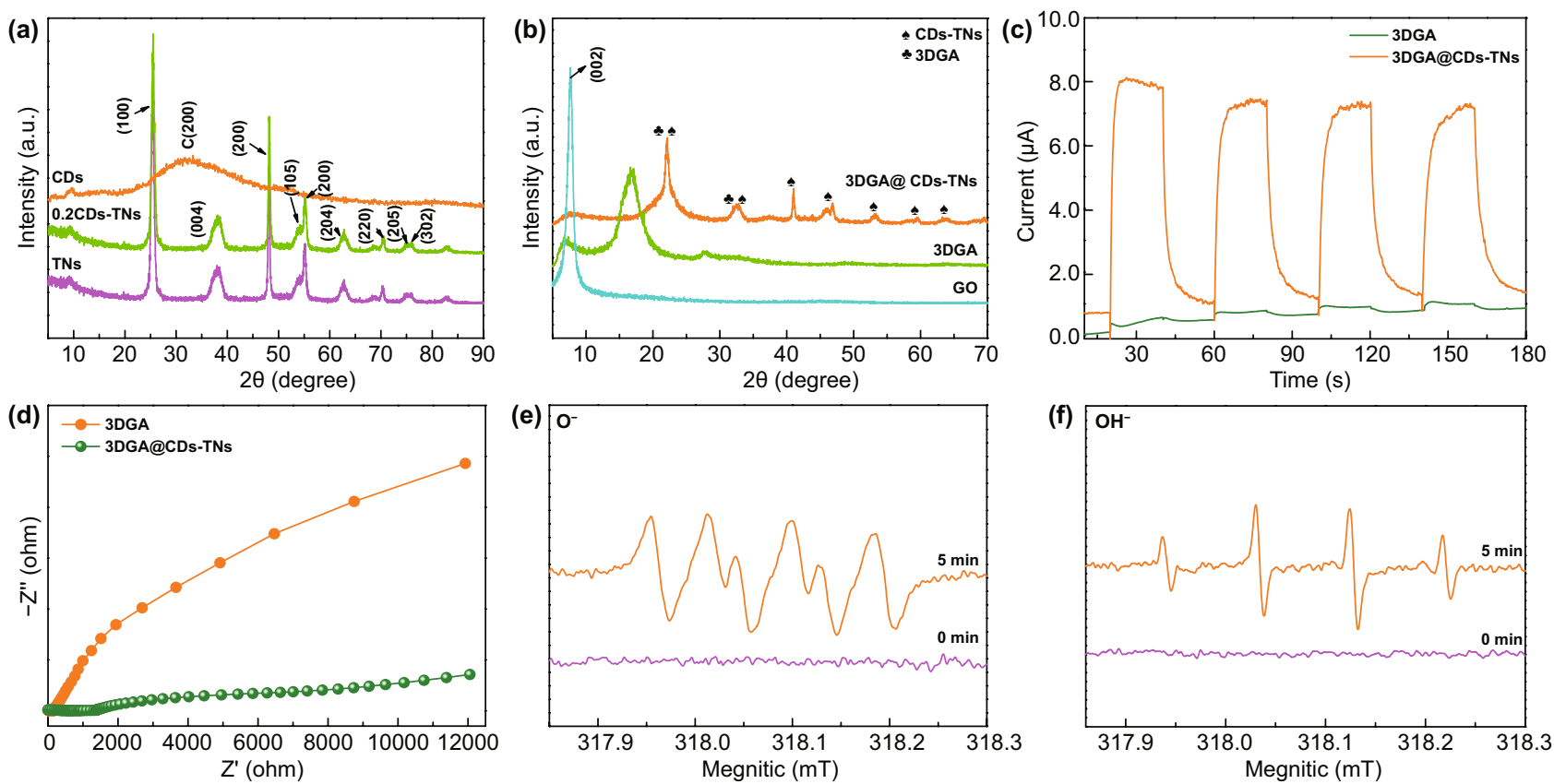

Fig. 3 XRD patterns of samples: a CDs, TNs, and CDs-TNs, b GO, 3DGA, and 3DGA@CDs-TNs photocatalyst. c EIS curves and d transient photocurrent densities for 3DGA@CDs-TNs photocatalyst under intermittent visible light irradiation. ESR spin-trapping profiles for e DMOP- $\cdot \mathrm{O}_{2}{ }^{-}$and $\mathbf{f} \cdot \mathrm{OH}$ generation over 3DGA@CDs-TNs photocatalyst

Transient photocurrent response and EIS measurements reveal the charge transfer ability of 3DGA@CDs-TNs photocatalyst [56]. From Fig. 3c, the current intensity for 3DGA@CDs-TNs photocatalyst is significantly increased after introducing CDs-TNs. Obviously, a narrower arc radius (Fig. 3d) is observed for 3DGA@CDs-TNs photocatalyst under visible light simulation with respect to that of 3DGA, which demonstrates that the loading of CDs-TNs can facilitate charge transport and separation, resulting in a superior degradation capacity of 3DGA@ CDs-TNs photocatalyst. Moreover, CDs/TNs show a dramatically enhanced photocurrent response and a smaller semicircle diameter (Fig. S3a, b), revealing that CDs can accelerate the migration rate of $\mathrm{e}^{-}-\mathrm{h}^{+}$pairs, thereby contributing to the remarkable photodegradation performance. Additionally, electron spin resonance (ESR) technique was also carried out for detecting DMPO- ${ }_{2}^{-}$and DMPO-OH. As illustrated in Fig. 3e, f, no signal of DMPO-.OH is monitored under the darkness in the existence of 3DGA@ CDs-TNs photocatalyst. After irradiating for $5 \mathrm{~min}$, four typical peaks of $\cdot \mathrm{OH}$ are observed. Similarly, no characteristic peaks of $\cdot \mathrm{O}_{2}{ }^{-}$are observed in the absence of visible light. Since irradiated to visible light, four obvious signals of $\cdot \mathrm{O}_{2}{ }^{-}$can be captured, which verify that the $\mathrm{H}_{2} \mathrm{O}$ and $\mathrm{O}_{2}$ in the reaction system can be converted into $\cdot \mathrm{OH}$ and $\cdot \mathrm{O}_{2}{ }^{-}$by catalyst under visible light irradiation, thus degrading hazardous pollutants directly.

\subsection{Degradation Performance of TENG, 3D3DGA@ CDs-TNs and TENG/3DGA@CDs-TNs}

The degradation performance of TENG and photocatalyst are assessed by decomposing two representative structure pollutants: azo dye-DB and triphenylmethane dye-BG (Fig. 4). The characteristic absorbance of BG solution changed via different approach (TENG, 3DGA@CDs-TNs photocatalyst and TENG/3DGA@CDs-TNs) at different reaction times is shown in Fig. $4 \mathrm{a}-\mathrm{c}$. The results display the absorption intensities of BG decrease more rapidly with increasing the reaction time. The absorption peak at $624 \mathrm{~nm}$ seems to be completely disappeared with degradation time extending, verifying that BG is almost removed by TENG, 3DGA@CDs-TNs photocatalyst, and TENG/3DGA@CDsTNs, respectively. Meanwhile, the peak at about $425 \mathrm{~nm}$ increases firstly and then decreases, revealing that organic fragments are decomposed to small molecules. Significantly, 

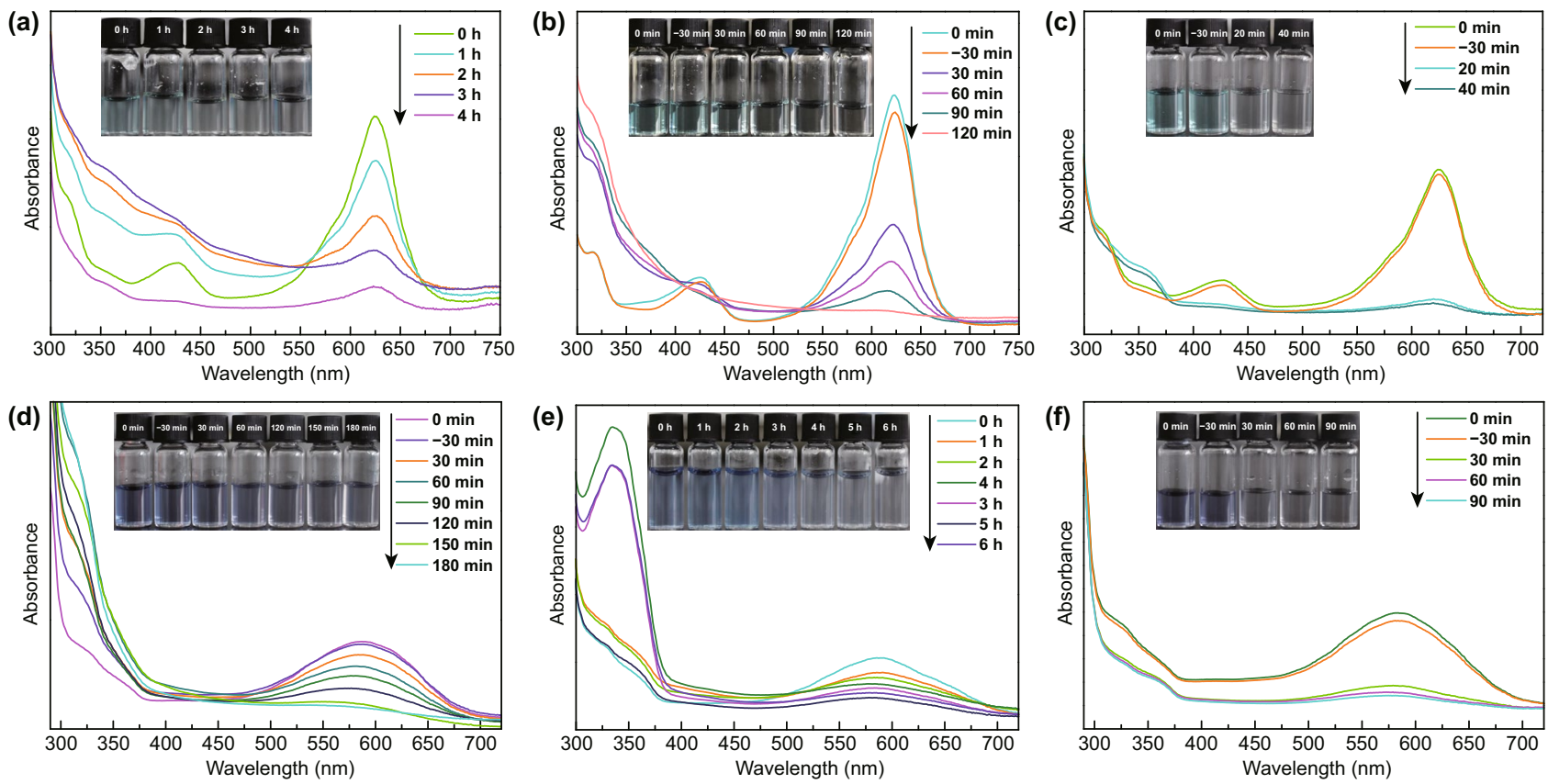

Fig. 4 Changes of the characteristic absorption after degradation by 3DGA@CDs-TNs photocatalyst, TENG, and TENG/3DGA@CDs-TNs of a-c BG and photographs of residual BG solution (inset); d-f DB and photographs of residual DB solution (inset)

in the case of TENG/3DGA@CDs-TNs experiment, the absorption peak dips quickly in the first 20 min of degradation and is flat after $40 \mathrm{~min}$, highlighting that BG degradation efficiency highly depends on the presence of TENG and 3DGA@CDs-TNs photocatalyst. The inset photographs in Fig. $4 \mathrm{a}-\mathrm{c}$ indicate that $\mathrm{BG}$ aqueous solution is almost colorless by degradation. The spectrum resulting from the three methods of DB are portrayed in Fig. 4d-f. With reaction time prolong, the main peak at about $586 \mathrm{~nm}$ decreases. The color of DB solution almost disappears after decomposition, indicating the completely destruction of $\mathrm{DB}$ conjugated structure.

To directly compare the degradation rates of pollutants over the three methods, the removal efficiency and the kinetics of degradation are also concluded (Fig. 5a-d). For the TENG experiment, the results show that $59.59 \%$ of BG can be degraded effectively under the rotator speed of $300 \mathrm{r}$ $\min ^{-1}$. Moreover, 3DGA@CDs-TNs photocatalyst exhibits the removal efficiency of $81.66 \%$ (BG) after $2 \mathrm{~h}$ visible light irradiation. Dramatically, in the existence of TENG and 3DGA@ CDs-TNs photocatalyst, remarkable enhancements $(88.26 \%$, $40 \mathrm{~min}$ ) in the decomposition of BG are observed. TENG, 3DGA@CDs-TNs photocatalyst, and TENG/3DGA@CDsTNs display favorable effect on the decomposition of $\mathrm{DB}$, in which degradation rates are $59.2 \%(6 \mathrm{~h}), 73.23 \%(3 \mathrm{~h})$, and $89.6 \%$ (1.5 h), respectively (Table S2). As shown in Fig. 5a, b, the combination of TENG and 3DGA@CDs-TNs photocatalyst results in a higher capacity of pollutants degradation. This phenomenon is ascribed to that TENG provides bias potentials and current which can build an electric field and accelerate the separation and transfer of carriers, leading to an increased degradation efficiency. Furthermore, 3DGA exhibits the merits of electron collection and transportation and can efficiently hinder the recombination of photogenerated charges in hybrid process. The combination of TENG and 3DGA@CDs-TNs photocatalyst endows the TENG/3DGA@CDs-TNs system an interesting candidate for pollutants degradation in sunlight and ocean condition.

In addition, the kinetics of degradation under different conditions are expressed in the following equation:

$\ln \left(C_{t} / C_{0}\right)=-k t$

where $C_{0}, C_{t}$, and $k$ are the initial equilibrium concentration, actual concentration of dyes at reaction time $t$, and rate constants, respectively. The relationship between $\ln \left(C_{t} / C_{0}\right)$ and t over TENG, 3DGA@CDs-TNs photocatalyst, and TENG/3DGA@CDs-TNs is plotted in Fig. 5c, d. From the result, it can be seen that the degradation of DB and BG follows the pseudo-first-order kinetics. The degradation kinetic 

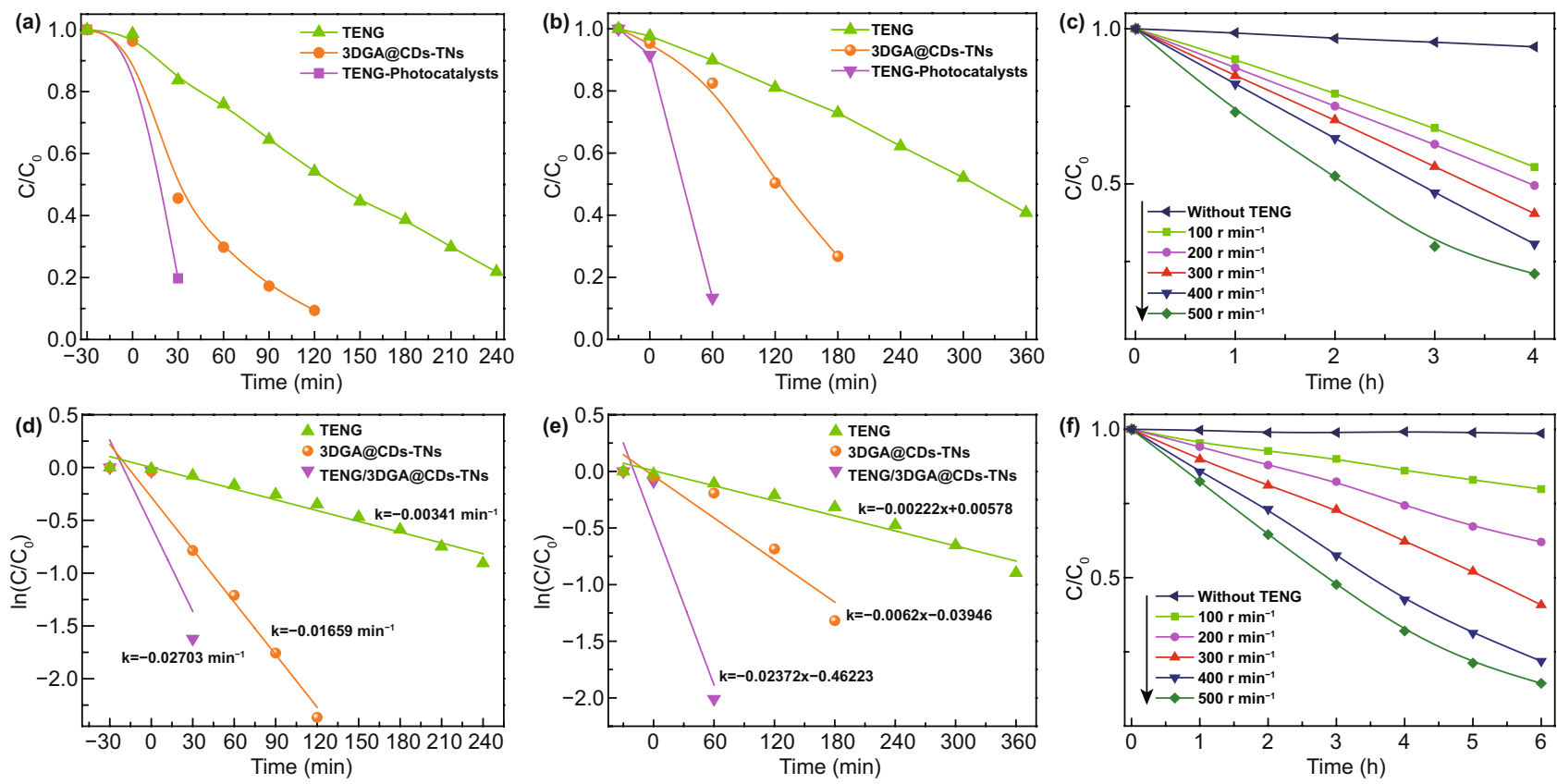

Fig. 5 Degradation and degradation kinetics curves of BG (a, b) and DB (c, d) for 3DGA@CDs-TNs photocatalyst, TENG, and TENG/3DGA@CDs-TNs. Degradation of e BG, $\mathbf{f}$ DB by TENG with different rotation rates

parameters of TENG, 3DGA@CDs-TNs photocatalyst and TENG/3DGA@CDs-TNs are summarized in Tables S3 and S4. It can be seen that the TENG/3DGA@CDs-TNs system has the highest reaction rate constants of 0.01011 and 0.01338 for BG and DB dyes, respectively. The results illustrate the TENG-3DGA@CDs-TNs system achieves the highest degradation of pollutants compared to the TENG and 3DCA@CDs-TNs photocatalyst due to superior cooperation of photoelectron.
Figure 5e, f demonstrates the removal efficiency of the two pollutants at different rotation rates. As shown in Fig. 5e, f, $500 \mathrm{r} \mathrm{min}^{-1}$ has the fastest degradation rate, while $100 \mathrm{r}$ $\min ^{-1}$ has the slowest. Concretely, as the speed rate increases from 100 to $500 \mathrm{r} \mathrm{min}^{-1}$, the degradation rates for DB and BG increase from $20.07 \%, 40.61 \%$ to $77.8 \%$ and $85.55 \%$, respectively (Fig. S4a, b). The increased degradation rates

Table 1 Comparison of pollutants degradation over different techniques

\begin{tabular}{|c|c|c|c|c|c|}
\hline Technique & Light source & [Pollutants] $\left(\mathrm{mg} \mathrm{L}^{-1}\right)$ & [Catalyst] $\left(\mathrm{g} \mathrm{L}^{-1}\right)$ & Removal (\%) & Refs. \\
\hline Photo & Xe300W & Congred, 50 & 0.2 & $\sim 90(1 \mathrm{~h})$ & [7] \\
\hline Photo & $40 \mathrm{~W}$ UV & Congred, 30 & 0.5 & $78(2 \mathrm{~h})$ & [9] \\
\hline Photo & Visible 300 & RhodamineB, 10 & 0.5 & $\sim 97(2 \mathrm{~h})$ & {$[10]$} \\
\hline Photo & two EDMLs & RhodamineB, 10 & 1 & $\sim 90(1 \mathrm{~h})$ & [11] \\
\hline Fenton & - & 4-nitrophenol, 10 & - & $91(1 \mathrm{~h})$ & {$[20]$} \\
\hline Fenton & - & Orange II, 30 & - & $90(20 \mathrm{~min})$ & {$[21]$} \\
\hline Fenton & - & Phenol, 30 & - & $80(50 \mathrm{~min})$ & {$[22]$} \\
\hline Photo-Fenton & - & Phenol, 30 & 1 & $90(50 \mathrm{~min})$ & {$[22]$} \\
\hline Photo-Fenton & Xe350W & Methylene B, 30 & $1 \mathrm{~L}$ & $88(50 \mathrm{~min})$ & {$[23]$} \\
\hline Photo-Fenton & Xe350W & Ibuprofen, 20 & 0.5 & $90(60 \mathrm{~min})$ & [24] \\
\hline Photo-Fenton & Xe300W & Tetracycline, 20 DB, & 0.4 & $62.7(40 \mathrm{~min})$ & {$[25]$} \\
\hline \multirow[t]{2}{*}{ TENG-photo } & Xe300W & $20 \mathrm{BG}, 5$ & 0.5 & $89.5(1.5 \mathrm{~h})$ & This work \\
\hline & Visible $500 \mathrm{~W}$ & & & 88.26 (40 min) & This work \\
\hline
\end{tabular}


(a)

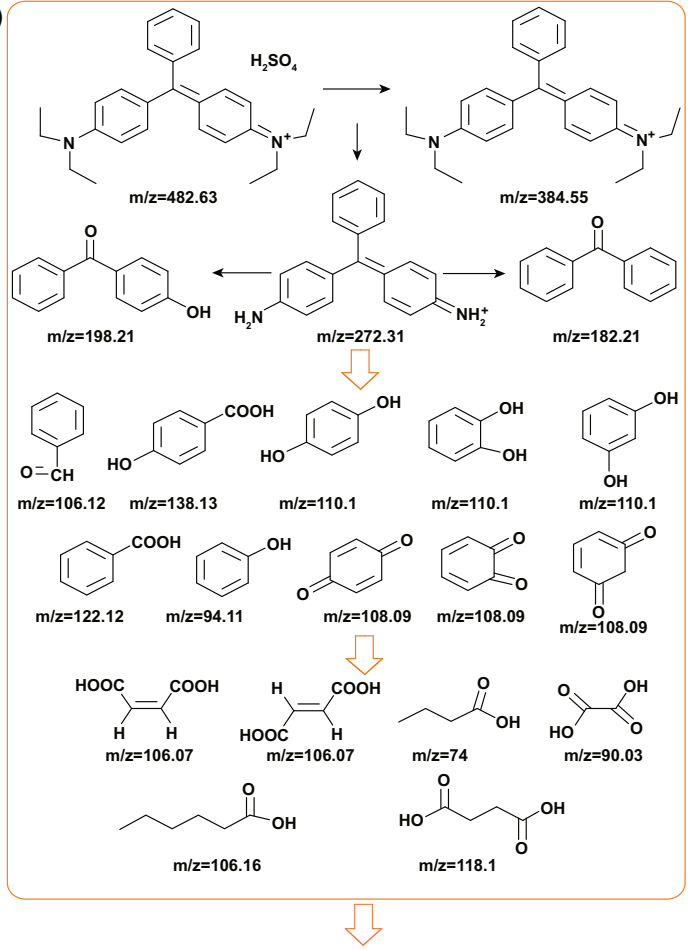

$\mathrm{CO}_{2}, \mathrm{H}_{2} \mathrm{O}$ and small molecules (b)

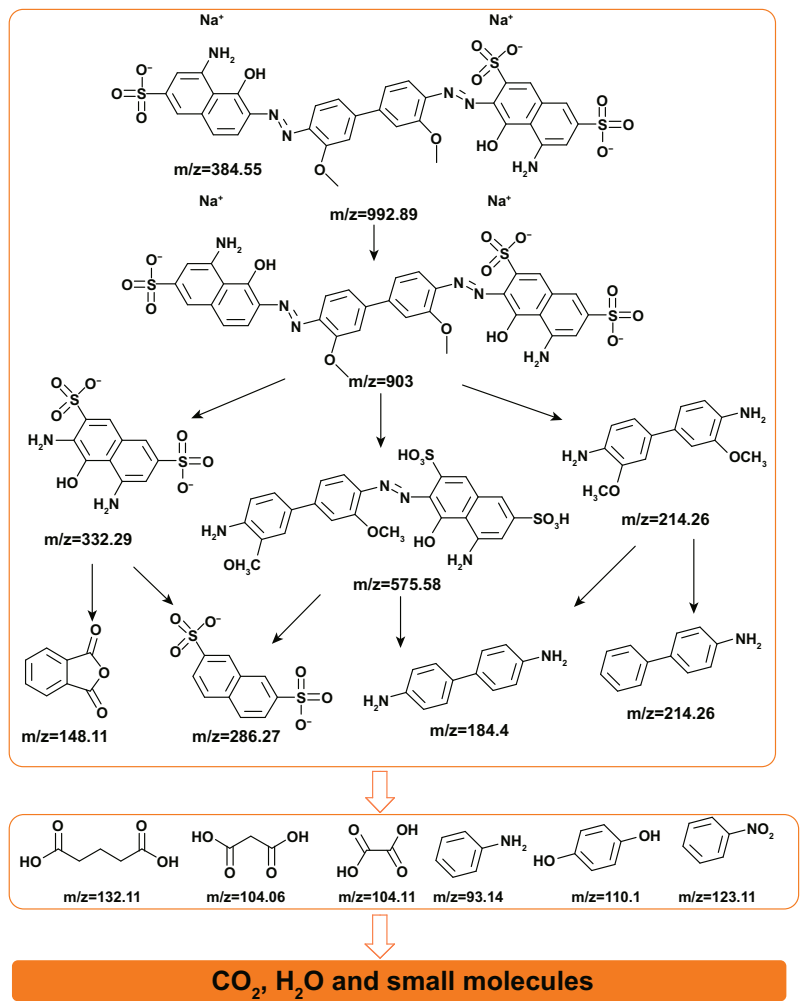

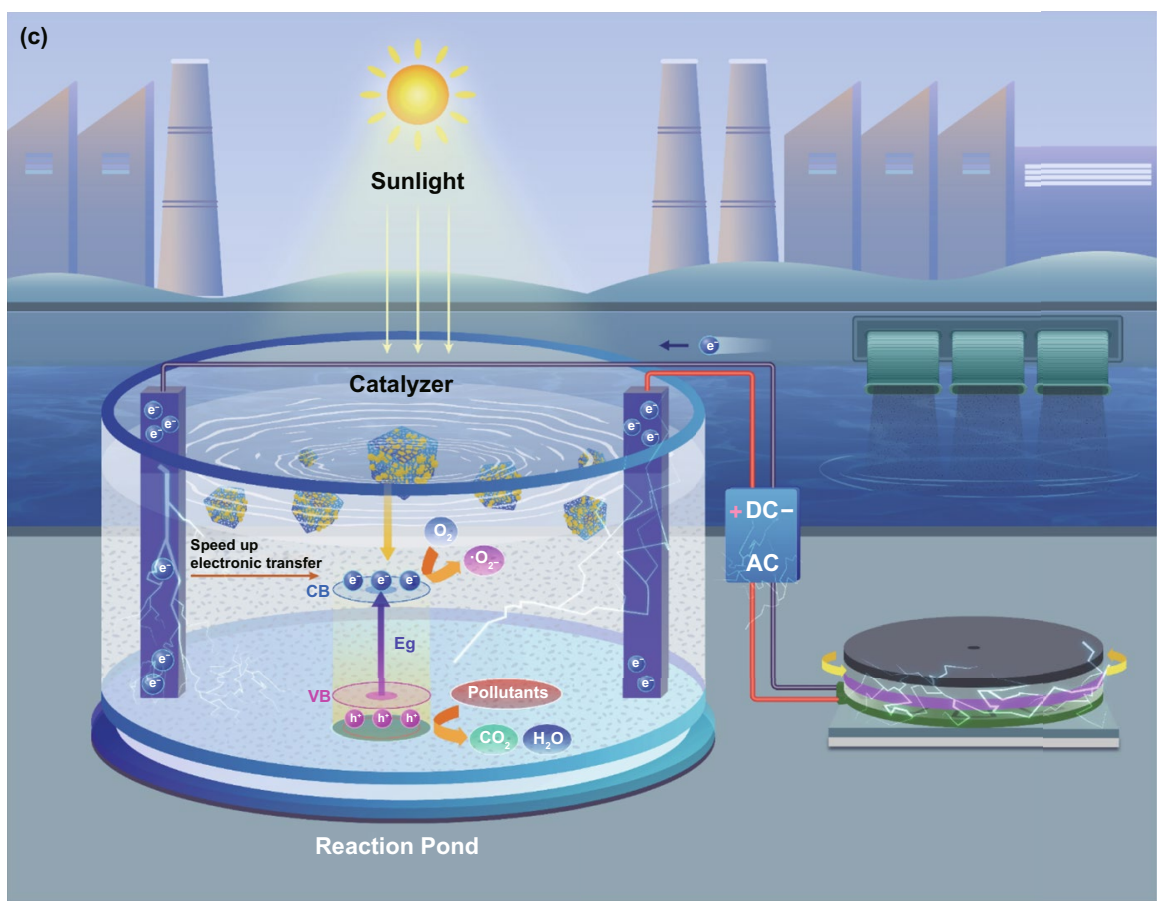

Fig. 6 Possible pathways for the degradation of $\mathbf{a}$ BG and $\mathbf{b}$ DB over TENG/3DGA@CDs-TNs photocatalysts under visible light irradiation. $\mathbf{c}$ Schematic showing the degradation mechanism of TENG/3DGA@CDs-TNs 
illustrate that the higher rotation rate enhances the degradation capability of TENG.

In Table 1, the degradation performance of TENG/3DGA@CDs-TNs is compared with other techniques reported previously. Obviously, taking into account the different experimental parameters, TENG/3DGA@CDsTNs exhibits outstanding degradation activity among these methods.

\subsection{Degradation Pathway and Mechanism}

Notwithstanding the pollutants can be removed by TENG and 3DGA@CDs-TNs photocatalyst, hazardous fragments produced during the reaction process may also bring about environmental compounds [57]. Hence, LC-MS technique was employed to identify the transformation intermediates and final products of DB and BG. Possible small molecules and relative details regarding $\mathrm{DB}$ and $\mathrm{BG}$ are listed in Tables S5 and S6. According to the LC-MS results, fifteen intermediates of DB are deduced. As shown in Fig. 6a, product with $m / z=905$ is derived from the initial DB, fragments with $m / z=332.29$ and $m / z=214.26$ are further produced by the breaking of $-\mathrm{N}=\mathrm{N}-$, and $m / z=575.58$ is assign to the cleavage of $-\mathrm{C}-\mathrm{C}-$ bond. And $m / z=575.58$ can form $m / z=286.27$ and $m / z=184.24$ (benzidinel) via the breaking of $-\mathrm{N}=\mathrm{N}-$. Intermediates with $\mathrm{m} / \mathrm{z}=148.11$ (o-phthalic anhydride) and $m / z=214.26$ (4-aminobiphenyl hydrochloride) are owning to the oxidation of $m / z=332.29$ and 214.26, respectively. Contaminants with $\mathrm{m} / \mathrm{z}=93.14$, $m / z=110.1$, and $m / z=123.11$ can be attributed to the oxidation of $m / z=184.24$ and ring opening of $m / z=148.11$. Subsequently, products with $m / z=104.06, m / z=104.11$, and $\mathrm{m} / \mathrm{z}=132.11$ are resulted from the mineralization of benzene ring. Based on the intermediates and final products analysis, the feasible decomposition pathway for DB is deduced.

Furthermore, the plausible removal pathway for BG is also proposed (Fig. 6b). Briefly, the ethyl group on the maternal BG is firstly attracted by free radicals, transforming the compound of $m / z=272.31$. Moreover, fragments $m / z=198.21, m / z=182.21$, and $m / z=106.12$ are assigned to the cleavage of $\mathrm{m} / z=272.31$. Then, the opening ring and mineralization reaction is emerged, and small molecules are produced by the breakdown of BG and the $\mathrm{N}$-de-ethylated fragments.
In summary, the initial subtracts and unstable intermediates may be decomposed to $\mathrm{CO}_{2}, \mathrm{H}_{2} \mathrm{O}$, and other small molecules.

Based on the principle of catalysis and our experiments, the photoinduced charge generation, separation, and transport in pollutants degradation as well as the mechanism of photoelectric catalysis are proposed (Fig. 6c). On the one hand, under visible light excitation, CDs can absorb visible light and emit UV light, which excites TNs to produce electron-hole pairs. The electrons can transit toward 3DGA and shuttle efficiently from CDs-TNs catalyst to aerogel, thus prolonging charge carrier lifetime and inhibiting the recombination of electron-hole pairs. On the other hand, when TENG is applied to the degradation process, the current of TENG can enhance the concentration of carriers because of the injection of electrons. Meanwhile, the applied bias can accelerate the separation of electron-hole pairs and hinder the rapid decay of photocurrent. In other words, the visible light simulation is used to induce the excitation within the semiconductor, while the applied bias, as an external driving force, is employed to promote the continuous separation of electron-hole pairs. Then, the electrons consume the superficial oxygen to generate superoxide $\left(\cdot \mathrm{O}_{2}{ }^{-}\right)$, which is taken part in the degradation process. Moreover, the holes of photocatalyst combine with $\mathrm{H}_{2} \mathrm{O}$ or $\mathrm{OH}^{-}$to form $\cdot \mathrm{OH}$, which directly decompose the organic pollutants.

\section{Conclusion}

This study develops a novel TENG coupled with 3DGA@ CDs-TNs system. The designed TENG can yield a high voltage of $\sim 510 \mathrm{~V}$ and a high power density of $25.48 \mathrm{~W} \mathrm{~m}^{-2}$. The realization of synergetic photoelectric catalysis further increases the efficiency of photocatalyst in dyes degradation. The hybrid degradation system presents 6.65 -fold and 5.74-fold enhancements for photoelectrocatalytic degradation of BG and DB compared to that of TENG. Besides, the kinetics of degradation of BG and DB over TENG/3DGA@ CDs also exhibit 2.81-time and 1.99-time higher than those of 3DGA@CDs-TNs photocatalyst. The enhancement can be ascribed to the strong interaction TENG and 3DGA@ CDs-TNs photocatalyst, which is due to that the TENG can provide photogenerated electrons and applied bias, promoting the separation and transfer of electrons and holes. Based on the results of LC-MS, the feasible degradation pathways 
for BG and DB are also clarified. This compelling strategy of converting mechanical energy and solar into sustainable energy sparks an inspiration in designing environmentalfriendly photoelectrocatalytic system with excellent wastewater remediation performance.

Acknowledgements This study was financially supported by the National Key R\&D Program of China (Grant Nos. 2016YFA0202704 and 2019YFA0706900); the Beijing Municipal Natural Science Foundation (Grant No. 2212052); the China Postdoctoral Science Foundation (Grant No. 2019T120390); and the Jiangsu Planned Projects for Postdoctoral research funds (Grant No. 2018K018A). The authors would also like to thank Shiyanjia laboratory for their professional tests support.

CRediT authorship contribution statement S. Shen, K. Dong, and Z.L. Wang conceived and designed the research. S. Shen and K. Dong conducted the experiment and characterization. Y. Jia, L. Ma, F. Sheng, C. Li, T. Wang, and C. Ning helped the material synthesis and data processing. H. Wang, J. Fu, K. Dong, and Z.L. Wang supervised, reviewed, and edited the research. All authors discussed and analyzed the data. S. Shen, K. Dong, and Z.L. Wang co-wrote the paper.

Open Access This article is licensed under a Creative Commons Attribution 4.0 International License, which permits use, sharing, adaptation, distribution and reproduction in any medium or format, as long as you give appropriate credit to the original author(s) and the source, provide a link to the Creative Commons licence, and indicate if changes were made. The images or other third party material in this article are included in the article's Creative Commons licence, unless indicated otherwise in a credit line to the material. If material is not included in the article's Creative Commons licence and your intended use is not permitted by statutory regulation or exceeds the permitted use, you will need to obtain permission directly from the copyright holder. To view a copy of this licence, visit http://creativecommons.org/licenses/by/4.0/.

Supplementary Information The online version contains supplementary material available at https://doi.org/10.1007/ s40820-021-00695-3.

\section{References}

1. J. Hu, P. Zhang, W. An, L. Liu, Y. Liang et al., In-situ Fedoped $\mathrm{g}-\mathrm{C}_{3} \mathrm{~N}_{4}$ heterogeneous catalyst via photocatalysisfenton reaction with enriched photocatalytic performance for removal of complex wastewater. Appl. Catal. B-Environ. 245, 130-142 (2019). https://doi.org/10.1016/j.apcatb.2018.12.029

2. B. Tian, Y. Wu, G. Lu, Metal-free plasmonic boron phosphide/graphitic carbon nitride with core-shell structure photocatalysts for overall water splitting. Appl. Catal. B-Environ. 280, 119410 (2021). https://doi.org/10.1016/j.apcatb. 2020.119410

3. P. Yang, R. Wang, H. Tao, Y. Zhang, M.-M. Titirici et al., Cobalt nitride anchored on nitrogen-rich carbons for efficient carbon dioxide reduction with visible light. Appl. Catal. B-Environ. 280, 119454 (2021). https://doi.org/10.1016/j. apcatb.2020.119454

4. H. Xu, Y. Jiang, X. Yang, F. Li, A. Li et al., Fabricating carbon quantum dots doped $\mathrm{ZnIn}_{2} \mathrm{~S}_{4}$ nanoflower composites with broad spectrum and enhanced photocatalytic tetracycline hydrochloride degradation. Mater. Res. Bull. 97, 158-168 (2018). https://doi.org/10.1016/j.materresbull.2017.09.004

5. T. Xu, D. Wang, L. Dong, H. Shen, W. Lu et al., Graphitic carbon nitride co-modified by zinc phthalocyanine and graphene quantum dots for the efficient photocatalytic degradation of refractory contaminants. Appl. Catal. B-Environ. 244, 96-106 (2019). https://doi.org/10.1016/j.apcatb.2018.11.049

6. J. Shi, F. Chen, L. Hou, G. Li, Y. Li et al., Eosin y bidentately bridged on $\mathrm{UIO}_{66} \mathrm{NH}_{2}$ by solvothermal treatment towards enhanced visible-light-driven photocatalytic $\mathrm{H}_{2}$ production. Appl. Catal. B-Environ. 280, 119385 (2021). https://doi.org/ 10.1016/j.apcatb.2020.119385

7. S. Shen, J.J. Fu, H.B. Wang, Unravelling the favorable photocatalystic effect of hydrogenation process on the novel g- $\mathrm{C}_{3} \mathrm{~N}_{4}$ $-\mathrm{TiO}_{2}$ catalysts for water purification. Diam. Relat. Mater. 114, 108292 (2021). https://doi.org/10.1016/j.diamond.2021. 108292

8. S. Xin, G. Liu, X. Ma, J. Gong, B. Ma et al., High efficiency heterogeneous fenton-like catalyst biochar modified $\mathrm{CuFeO}_{2}$ for the degradation of tetracycline: Economical synthesis, catalytic performance and mechanism. Appl. Catal. B-Environ. 280, 119386 (2021). https://doi.org/10.1016/j.apcatb.2020. 119386

9. Y. Li, B. Huang, Y. Sun, M. Luo, Y. Yang et al., Multimetal borides nanochains as efficient electrocatalysts for overall water splitting. Small 15, 1804212 (2019). https://doi.org/10. 1002/smll.201804212

10. D.Y. Yoon, J.H. Park, H.C. Kang, P.S. Kim, I.S. Nam et al., Denox performance of $\mathrm{Ag} / \mathrm{Al}_{2} \mathrm{O}_{3}$ catalyst by n-dodecane: Effect of calcination temperature. Appl. Catal. B-Environ. 101, 275-282 (2011). https://doi.org/10.1016/j.apcatb.2010. 09.028

11. Y. Wang, M. Zhang, H. Yu, Y. Zuo, J. Gao et al., Facile fabrication of $\mathrm{Ag} / \mathrm{graphene}$ oxide/ $/ \mathrm{TiO}_{2}$ nanorod array as a powerful substrate for photocatalytic degradation and surface-enhanced raman scattering detection. Appl. Catal. B-Environ. 252, 174186 (2019). https://doi.org/10.1016/j.apcatb.2019.03.084

12. J. Wang, G. Wang, B. Cheng, J. Yu, J. Fan, Sulfur-doped g- $\mathrm{C}_{3} \mathrm{~N}_{4} / \mathrm{TiO}_{2}$ s-scheme heterojunction photocatalyst for congo red photodegradation. Chinese J. Catal. 42(1), 56-68 (2021). https://doi.org/10.1016/s1872-2067(20)63634-8

13. M.Y. Rizal, R. Saleh, S.P. Prakoso, A. Taufik, S. Yin, Ultraviolet- and visible-light photocatalytic and sonophotocatalytic activities toward congo red degradation using $\mathrm{Ag} / \mathrm{Mn}_{3} \mathrm{O}_{4}$ 
nanocomposites. Mater. Sci. Semicon. Proc. 121, 105371 (2021). https://doi.org/10.1016/j.mssp.2020.105371

14. X. Shan, G. Ge, Z. Zhao, Facile and scalable fabrication of porous $\mathrm{g}-\mathrm{C}_{3} \mathrm{~N}_{4}$ nanosheets with nitrogen defects and oxygendoping for synergistically promoted visible light photocatalytic $\mathrm{H}_{2}$ evolution. Energy Technol. 7(5), 1800886 (2019). https://doi.org/10.1002/ente.201800886

15. Z. Liu, Z. Wang, S. Qing, N. Xue, S. Jia, Improving methane selectivity of photo-induced $\mathrm{CO}_{2}$ reduction on carbon dots through modification of nitrogen-containing groups and graphitization. Appl. Catal. B-Environ. 223, 86-92 (2018). https://doi.org/10.1016/j.apcatb.2018.03.045

16. F. Li, M. Zhang, D. Benetti, L. Shi, L.V. Besteiro et al., "Green", gradient multi-shell $\mathrm{CuInSe}_{2} /\left(\mathrm{CuInSeXS}_{1-\mathrm{x})(5)} /\right.$ $\mathrm{CuInS}_{2}$ quantum dots for photo-electrochemical hydrogen generation. Appl. Catal. B-Environ. 280, 119402 (2021). https:// doi.org/10.1016/j.apcatb.2020.119402

17. J. Su, L. Zhu, G. Chen, Ultrasmall graphitic carbon nitride quantum dots decorated self-organized $\mathrm{TiO}_{2}$ nanotube arrays with highly efficient photoelectrochemical activity. Appl. Catal. B-Environ. 186, 127-135 (2016). https://doi.org/10. 1016/j.apcatb.2015.12.050

18. Y. Zhang, M. Wu, Y. Wang, Y.H. Kwok, W. Pan et al., Fluorinated $\mathrm{TiO}_{2}$ coupling with alpha- $\mathrm{MnO}_{2}$ nanowires supported on different substrates for photocatalytic VOCs abatement under vacuum ultraviolet irradiation. Appl. Catal. B-Environ. 280, 119402 (2021). https://doi.org/10.1016/j.apcatb.2020.119388

19. B. Yang, J. Xu, D. Bin, J. Wang, J. Zhao et al., Amorphous phosphatized ruthenium-iron bimetallic nanoclusters with Pt-like activity for hydrogen evolution reaction. Appl. Catal. B-Environ. 283, 119583 (2021). https://doi.org/10.1016/j. apcatb.2020.119583

20. D. Xing, Z. Cui, Y. Liu, Z. Wang, P. Wang et al., Twodimensional Pi-d conjugated metal-organic framework Fe${ }_{3}$ (hexaiminotriphenylene) $_{(2)}$ as a photo-fenton like catalyst for highly efficient degradation of antibiotics. Appl. Catal. B-Environ. 290, 120029 (2021). https://doi.org/10.1016/j. apcatb.2021.120029

21. M.J. Bai, X.-Y. Huang, H. Yin, D.-L. Nu, J. Wan, Fe-doped graphitic carbon nitride for methylene blue degradation with visible-light. J. Nanosci. Nanotechn. 21(11), 5698-5706 (2021). https://doi.org/10.1166/jnn.2021.19487

22. Z. Lu, X. Cao, H. Wei, W. Huo, Q. Wang et al., Strong enhancement effect of bisulfite on MIL- ${ }_{68}(\mathrm{Fe})$-catalyzed fenton-like reaction for organic pollutants degradation. Appl. Surface Sci. 542, 148631 (2021). https://doi.org/10.1016/j. apsusc. 2020.148631

23. P. Wu, C. Zhou, Y. Li, M. Zhang, P. Tao et al., Flower-like feooh hybridized with carbon quantum dots for efficient photofenton degradation of organic pollutants. Appl. Surface Sci. 540, 148362 (2021). https://doi.org/10.1016/j.apsusc. 2020. 148362

24. A. Hq, A. Gy, A. Qh, A. Yn, A. Cb et al., Ingenious control of adsorbed oxygen species to construct dual reaction centers [emailprotected] photo-fenton catalyst with high-speed electron transmission channel for ppcps degradation. Appl. Catal.
B-Environ. 291, 120064 (2021). https://doi.org/10.1016/j. apcatb.2021.120064

25. X.X. Qiao, K. Yu, J.-Y. Xu, Y.-L. Cai, Y.-F. Li et al., Engineered nanoscale schwertmannites as fenton-like catalysts for highly efficient degradation of nitrophenols. Appl. Surface Sci. 548, 149248 (2021). https://doi.org/10.1016/j.apsusc. 2021. 149248

26. S.G. Han, D.-D. Ma, S.-H. Zhou, K. Zhang, W.-B. Wei et al., Fluorine-tuned single-atom catalysts with dense surface Ni-N${ }_{4}$ sites on ultrathin carbon nanosheets for efficient $\mathrm{CO}_{2}$ electroreduction. Appl. Catal. B-Environ. 283, 119591 (2021). https://doi.org/10.1016/j.apcatb.2020.119591

27. S.S. Yi, Z.-Y. Wang, H.-M. Li, Z. Zafar, Z.-T. Zhang et al., Coupling effects of indium oxide layer on hematite enabling efficient photoelectrochemical water splitting. Appl. Catal. B-Environ. 283, 119649 (2021). https://doi.org/10.1016/j. apcatb.2020.119649

28. Y.J. Chung, C.-S. Yang, J.-T. Lee, G.H. Wu, J.M. Wu, Coupling effect of piezo-flexocatalytic hydrogen evolution with hybrid 1T-and 2H-phase few-layered mose nanosheets. Adv. Energy Mater. 10(42), 2002082 (2020). https://doi.org/10. 1002/aenm.202002082

29. P. Song, S. Kuang, N. Panwar, G. Yang, D.J.H. Tng et al., A self-powered implantable drug-delivery system using biokinetic energy. Adv. Mater. 29(11), 1605668 (2017). https:// doi.org/10.1002/adma.201605668

30. L. Ma, R. Wu, S. Liu, A. Patil, H. Gong et al., A machinefabricated 3D honeycomb-structured flame-retardant triboelectric fabric for fire escape and rescue. Adv. Mater. 32(38), 2003897 (2020). https://doi.org/10.1002/adma.202003897

31. K. Dong, J. Deng, W. Ding, A.C. Wang, P. Wang et al., Versatile core-sheath yarn for sustainable biomechanical energy harvesting and real-time human-interactive sensing. Adv. Energy Mater. 8(23), 1801111-1801114 (2018). https://doi. org/10.1002/aenm.201801114

32. X. Peng, K. Dong, C. Ye, Y. Jiang, Z.L. Wang, A breathable, biodegradable, antibacterial, and self-powered electronic skin based on all-nanofiber triboelectric nanogenerators. Sci. Adv. 6(26), 9624 (2020). https://doi.org/10.1126/sciadv. aba9624

33. K. Dong, Z. Wu, J. Deng, A. Wang, H. Zhou et al., A stretchable yarn embedded triboelectric nanogenerator as electronic skin for biomechanical energy harvesting and multifunctional pressure sensing. Adv. Mater. 30(43), e1804944 (2018). https://doi.org/10.1002/adma.201804944

34. Y.T. Lin, S.N. Lai, J.M. Wu, Simultaneous piezoelectrocatalytic hydrogen-evolution and degradation of water pollutants by quartz microrods@few-layered mos hierarchical heterostructures. Adv. Mater. 32(34), 2002875 (2020). https://doi. org/10.1002/adma.202002875

35. Y.C. Lai, Y.C. Hsiao, H.M. Wu, Z.L. Wang, Waterproof fabric-based multifunctional triboelectric nanogenerator for universally harvesting energy from raindrops, wind, and human motions and as self-powered sensors. Adv. Sci. 6(5), 1801883 (2019). https://doi.org/10.1002/advs.201801883 
36. Y.C. Lai, H.-M. Wu, H.C. Lin, C.L. Chang, H.H. Chou et al., Entirely, intrinsically, and autonomously self-healable, highly transparent, and superstretchable triboelectric nanogenerator for personal power sources and self-powered electronic skins. Adv. Funct. Mater. 29(40), 1904626 (2019). https://doi.org/ 10.1002/adfm.201904626

37. Z. Zhou, K. Chen, X. Li, S. Zhang, Y. Wu et al., Sign-tospeech translation using machine-learning-assisted stretchable sensor arrays. Nat. Electron. 3(9), 571-578 (2020). https://doi. org/10.1038/s41928-020-0428-6

38. G.R. Chen, Y.Z. Li, M. Bick, J. Chen, Smart textiles for electricity generation. Chem. Rev. 120(8), 3668-3720 (2020). https://doi.org/10.1021/acs.chemrev.9b00821

39. S. Zhang, M. Bick, X. Xiao, G. Chen, A. Nashalian et al., Leveraging triboelectric nanogenerators for bioengineering. Matter 4(3), 845-887 (2021). https://doi.org/10.1016/j.matt. 2021.01.006

40. L. Jin, X. Xiao, W. Deng, A. Nashalian, D. He et al., Manipulating relative permittivity for high-performance wearable triboelectric nanogenerators. Nano Lett. 20(9), 6404-6411 (2020). https://doi.org/10.1021/acs.nanolett.0c01987

41. Z.H. Zhou, L. Weng, T. Tat, A. Libanori, Z.M. Lin et al., Smart insole for robust wearable biomechanical energy harvesting in harsh environments. ACS Nano 14(10), 14126-14133 (2020). https://doi.org/10.1021/acsnano.0c06949

42. S.W. Chen, N. Wang, L. Ma, T. Li, M. Willander et al., Triboelectric nanogenerator for sustainable wastewater treatment via a self-powered electrochemical process. Adv. Energy Mater. 6(8), 9 (2016). https://doi.org/10.1002/aenm.201501778

43. L. Ma, M. Zhou, R. Wu, A. Patil, Z.L. Wang, Continuous and scalable manufacture of hybridized nano-micro triboelectric yarns for energy harvesting and signal sensing. ACS Nano 14(4), 4716-4726 (2020). https://doi.org/10.1021/acsnano. $0 \mathrm{c} 00524$

44. K. Dong, X. Peng, J. An, A.C. Wang, Z.L. Wang, Shape adaptable and highly resilient 3D braided triboelectric nanogenerators as e-textiles for power and sensing. Nat. Commun. 11(1), 2868 (2020). https://doi.org/10.1038/s41467-020-16642-6

45. K. Dong, X. Peng, Z.L. Wang, Fiber/fabric-based piezoelectric and triboelectric nanogenerators for flexible/stretchable and wearable electronics and artificial intelligence. Adv. Mater. 32(5), 1902549 (2020). https://doi.org/10.1002/adma.20190 2549

46. L. Ma, Q. Liu, R. Wu, Z. Meng, X.Y. Liu, From molecular reconstruction of mesoscopic functional conductive silk fibrous materials to remote respiration monitoring. Small 16(26), 2000203 (2020). https://doi.org/10.1002/smll.20200 0203

47. R. Wu, L. Ma, A.B. Patil, Z. Meng, X. Liu, Graphene decorated carbonized cellulose fabric for physiological signal monitoring and energy harvesting. J. Mater. Chem. A 8(25), 12665-12673 (2020). https://doi.org/10.1039/D0TA02221G

48. W. Tang, Y. Han, C.B. Han, C.Z. Gao, X. Cao et al., Selfpowered water splitting using flowing kinetic energy. Adv. Mater. 27(2), 272-276 (2015). https://doi.org/10.1002/adma. 201404071

49. K. Dong, Y.C. Wang, J. Deng, Y. Dai, S.L. Zhang et al., A highly stretchable and washable all-yarn-based self-charging knitting power textile composed of fiber triboelectric nanogenerators and supercapacitors. ACS Nano 11(9), 705317 (2017). https://doi.org/10.1021/acsnano.7b05317

50. Y.C. Wang, J.M. Wu, Effect of controlled oxygen vacancy on $\mathrm{H}_{2}$ production through the piezocatalysis and piezophototronics of ferroelectric $\mathrm{R}_{3} \mathrm{C} \mathrm{ZnSnO}_{3}$ nanowires. Adv. Funct. Mater. 30(5), 1907619 (2020). https://doi.org/10.1002/adfm.20190 7619

51. K. Han, J. Luo, Y. Feng, L. Xu, W. Tang et al., Self-powered electrocatalytic ammonia synthesis directly from air as driven by dual triboelectric nanogenerators. Energy Environ. Sci. 13(8), 2450-2458 (2020). https://doi.org/10.1039/d0ee01102a

52. A. Ahmed, I. Hassan, T. Ibn-Mohammed, H. Mostafa, I.M. Reaney et al., Environmental life cycle assessment and technoeconomic analysis of triboelectric nanogenerators. Energy Environ. Sci. 10(3), 653-671 (2017). https://doi.org/10.1039/ c7ee00158d

53. X. Han, Q. Kuang, M. Jin, Z. Xie, L. Zheng, Synthesis of titania nanosheets with a high percentage of exposed (001) facets and related photocatalytic properties. J. Am. Chem. Soc. 131(9), 3152-3153 (2009). https://doi.org/10.1021/ja8092373

54. M. Nawaz, W. Miran, J. Jang, D.S. Lee, One-step hydrothermal synthesis of porous $3 \mathrm{D}$ reduced graphene oxide $/ \mathrm{TiO}_{2}$ aerogel for carbamazepine photodegradation in aqueous solution. Appl. Catal. B-Environ. 203, 85-95 (2017). https://doi. org/10.1016/j.apcatb.2016.10.007

55. K. Dong, Y. Hu, J. Yang, S.-W. Kim, W. Hu et al., Smart textile triboelectric nanogenerators: current status and perspectives. MRS Bull. 46, 2021 (2021). https://doi.org/10.1557/ s43577-021-00123-2

56. D. Liu, D. Chen, N. Li, Q. Xu, H. Li et al., $\mathrm{ZIF}_{67}$ derived 3D hollow mesoporous crystalline $\mathrm{Co}_{3} \mathrm{O}_{4}$ wrapped by $2 \mathrm{D}$ g- $\mathrm{C}_{3} \mathrm{~N}_{4}$ nanosheets for photocatalytic removal of nitric oxide. Small 15(31), 1902291 (2019). https://doi.org/10.1002/smll.20190 2291

57. Z. Xie, Y. Feng, F. Wang, D. Chen, Q. Zhang, Construction of carbon dots modified $\mathrm{MoO}_{3} / g-\mathrm{C}_{3} \mathrm{~N}_{4}$ z-scheme photocatalyst with enhanced visible-light photocatalytic activity for the degradation of tetracycline. Appl. Catal. B-Environ. 229, 96-104 (2018). https://doi.org/10.1016/j.apcatb.2018.02.011 